\title{
Impact of remote ischemic preconditioning preceding coronary artery bypass grafting on inducing neuroprotection
}

\author{
Hrvoje Gasparovic, MD, PhD, ${ }^{a}$ Tomislav Kopjar, MD, PhD, ${ }^{a}$ Milan Rados, MD, PhD, ${ }^{b}$ \\ Alan Anticevic, MD, PhD, ${ }^{\mathrm{c}}$ Marko Rados, MD, PhD, ${ }^{d}$ Branko Malojcic, $\mathrm{MD}, \mathrm{PhD},{ }^{\mathrm{e}}$ \\ Visnja Ivancan, MD, PhD, ${ }^{a}$ Tea Fabijanic, MD,${ }^{a}$ Maja Cikes, MD, PhD, ${ }^{\mathrm{f}}$ Davor Milicic, MD, PhD, \\ Vladimir Gasparovic, $\mathrm{MD}, \mathrm{PhD},{ }^{\mathrm{f}}$ and Bojan Biocina, $\mathrm{MD}, \mathrm{PhD}^{\mathrm{a}}$
}

\section{ABSTRACT}

Background: Neurological complications after coronary artery bypass grafting $(\mathrm{CABG})$ reduce quality of life, increase mortality, and inflate resource utilization. The risk of postoperative neurological complications parallels the increasing risk burden of the contemporary patient population. We evaluated the efficacy of remote ischemic preconditioning (RIPC) on inducing neuroprotection.

Methods: Seventy patients undergoing first-time CABG were randomly assigned to RIPC or a sham procedure. Structural brain magnetic resonance imaging (MRI) was complemented with functional connectivity MRI to gain a whole-brain global connectivity analysis. Paired neurocognitive and MRI data were acquired pre- and postoperatively. The primary end point was a composite of new ischemic brain lesions and neurocognitive impairment. Secondary end points included brain connectivity profiles, pooled ischemic volumes, and individual components of the primary outcome. The Shapiro-Wilk test was used to determine whether a data set followed a normal distribution. The Fisher exact test was used to calculate the measures of association for categorical variables, whereas continuous data were tested with either the Mann-Whitney $U$ test or the Student $t$ test.

Results: There was no between-group difference in the incidence of the primary end point $(9[27 \%]$ in the RIPC group vs $8[24 \%]$ in the control group, odds ratio, 1.17 [95\% confidence interval, 0.34-4.06]; $P=1.0$ ). Although RIPC did not reduce the incidence of brain ischemia $(8 / 33[24 \%]$ vs $7 / 33[21 \%] ; P=1.0)$, the pooled ischemic volume was lower in the RIPC group (157 [interquartile range, 125-231] vs 777 [interquartile range, 564-965] $\left.\mathrm{mm}^{3} ; P=.004\right)$. Postoperative neurocognition was marginally superior in the RIPC group as evidenced by a lower absolute number of abnormal neurocognitive tests in the RIPC group (7/99 [7\%] vs 16/99 [16\%]; odds ratio, 0.40 [95\% confidence interval, 0.14-1.09]; $P=.074)$. Robust reductions of functional connectivity profiles for the associative thalamus were documented in both groups, irrespective of RIPC (RIPC group, $t=3.31 ; P<.01$; and the control group, $t=3.52 ; P<.01$ ).

Conclusions: Silent brain ischemia occurs frequently after CABG. RIPC did not reduce the incidence of the primary outcome. However, RIPC significantly

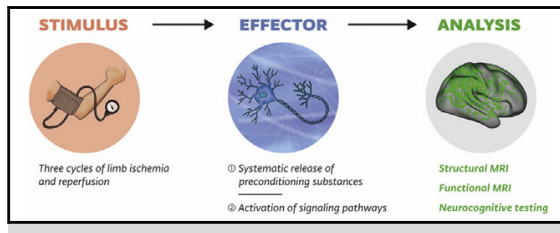

RIPCAGE study summary. Brain-wide postoperative reductions in associative thalamic functional connectivity (shown in green).

\section{Central Message}

Although RIPC did not reduce the incidence of new brain ischemia, it reduced its pooled volume. Surgery adversely affected global brain connectivity, with RIPC conferring no demonstrable protection.

\section{Perspective}

Ischemic preconditioning mobilizes the endogenous potential for protection against ischemia incurred by alternating periods of sublethal ischemia and reperfusion. Neuronal injury occurs in response to cardiac surgical interventions at the structural and functional levels. RIPC might have the potential to alleviate brain ischemic cellular damage and might thus provide an additional layer of neuroprotection, but this needs to be evaluated in a larger trial.

See Editorial Commentary page 1477.
From the ${ }^{\mathrm{a}}$ Department of Cardiac Surgery, ${ }^{\mathrm{d}}$ Department of Radiology, ${ }^{\mathrm{e}}$ Department of Neurology, ${ }^{\mathrm{f}}$ Departments of Cardiology and Internal Medicine, University Hospital Center Zagreb, ${ }^{b}$ Center of Excellence for Basic, Clinical and Translational Neuroscience, Croatian Institute for Brain Research, School of Medicine, University of Zagreb, Zagreb, Croatia; and ${ }^{\mathrm{c}}$ Departments of Psychiatry and Psychology, Yale University School of Medicine, New Haven, Conn.

The study was cofinanced by research grants from the University of Zagreb (grant 1.2.1.18) and the European Union through the European Regional Development Fund, Operational Program Competitiveness and Cohesion (KK.01.1.1.01.0007, CoRE - Neuro).
Received for publication March 28, 2018; revisions received Aug 17, 2018; accepted for publication Aug 29, 2018; available ahead of print Nov 15, 2018.

Address for reprints: Hrvoje Gasparovic, MD, PhD, Department of Cardiac Surgery, University Hospital Center Zagreb, Kispaticeva 12, 10000 Zagreb, Croatia (E-mail: hgasparovic@gmail.com).

$0022-5223 / \$ 36.00$

Copyright (C) 2018 Published by Elsevier Inc. on behalf of The American Association for Thoracic Surgery

https://doi.org/10.1016/j.jtcvs.2018.08.116 

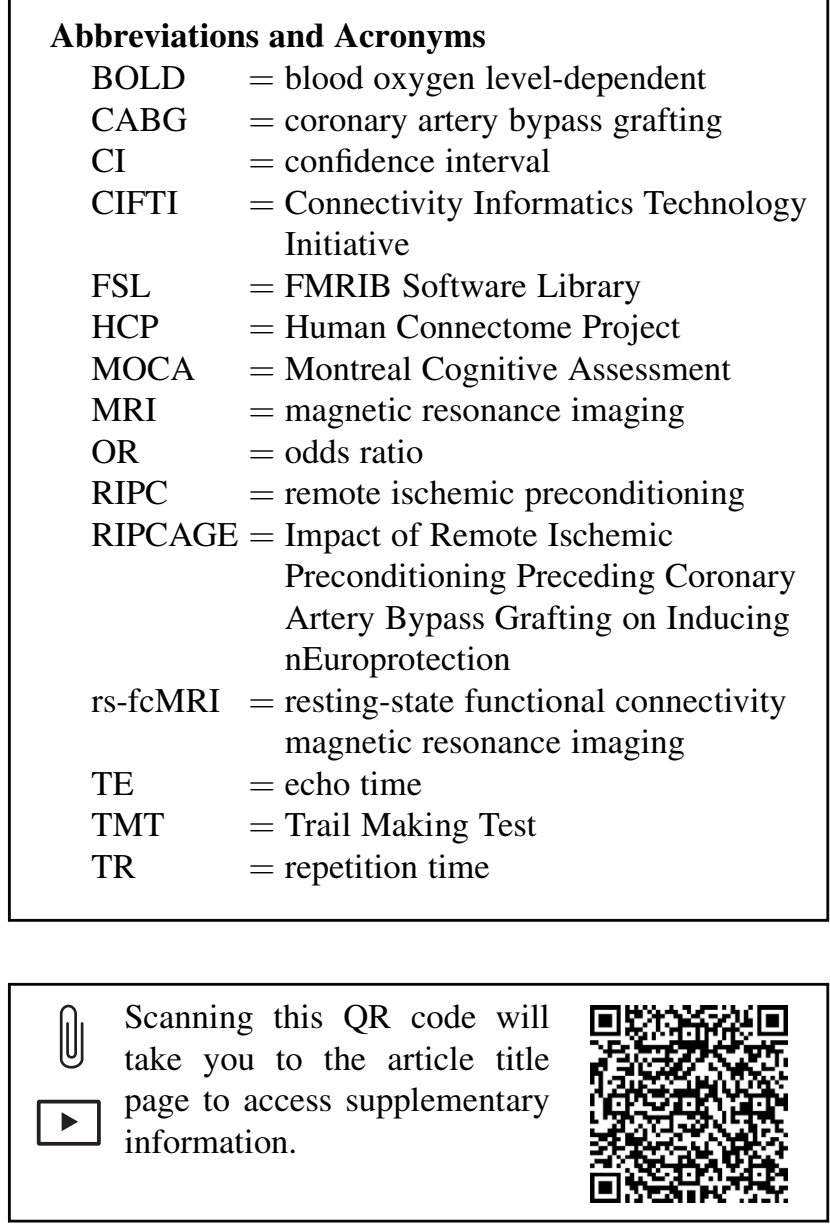

reduced the pooled volume of ischemic brain lesions. Surgery adversely affected global brain connectivity, with RIPC conferring no demonstrable protection. The association of RIPC with superior neurocognitive test scores failed to cross the threshold for significance. (J Thorac Cardiovasc Surg 2019;157:1466-76)

Neurological complications after cardiac surgery include stroke, delirium, and postoperative cognitive dysfunction. ${ }^{1-3}$ Although postoperative strokes are rare, their sequelae have profound effects on mortality and quality of life. Conversely, discrete impairments in attention, memory, and fine motor skills are often detected with sufficiently sensitive diagnostic tools. Remote ischemic preconditioning (RIPC) mobilizes the endogenous potential for protection against prolonged ischemia incurred by alternating periods of sublethal ischemia and reperfusion in tissues with high ischemic tolerance. ${ }^{4}$ Ischemic preconditioning has been shown to ameliorate excitotoxicity, which has been identified as one of the initial steps in the evolution of ischemic brain injury. ${ }^{5}$ Furthermore, neuronal resistance to ischemia is increased by synaptic and mitochondrial changes that occur in response to ischemic preconditioning-induced activation of key survival signaling pathways. ${ }^{5}$

Cardiopulmonary bypass incites a series of adverse events that might result in neuronal injury. Ischemia, inflammation, embolic events, cerebral edema, and hyperthermia act in concert to precipitate neurological complications. ${ }^{6}$ The continued inclusion of patients with progressively higher risk profiles into the contemporary cardiac surgical practice provides a strong impetus for defining targets that could improve outcomes. Although controversial data have been published on the efficacy of RIPC on myocardial protection in the cardiac surgical arena, RIPC remains an appealing strategy for inducing ischemic tolerance in this setting. ${ }^{7-9}$ To the best of our knowledge, this is the first trial to explore the translation of the RIPC concept onto induction of cerebral ischemic tolerance in the cardiac surgical population by examining structural ischemic changes as well as disturbances in brain-wide associative thalamic functional connectivity. We hypothesized that RIPC would lead to a reduction in the primary composite end point of new brain ischemia on magnetic resonance imaging (MRI) scans and new neurocognitive impairment.

\section{METHODS \\ Setting}

The study was conducted at the University Hospital Center Zagreb in Zagreb, Croatia. Patient enrollment started in March 2014 and was completed in December 2015. Details of the study design, eligibility, and inclusion and exclusion criteria have been published previously. ${ }^{10}$ The institutional review board evaluated and approved the conduct of this study (registration 380-59-10106-13-195/292). The Impact of Remote Ischemic Preconditioning Preceding Coronary Artery Bypass Grafting on Inducing nEuroprotection (RIPCAGE) trial has been registered at ClinicalTrials. gov (NCT02177981). All patients provided written informed consent.

\section{Study Participants}

Adult patients, aged 18 to 80 years, scheduled to undergo elective primary on-pump coronary artery bypass grafting (CABG) were screened for recruitment. Patient enrollment, data collection, and RIPC were done by trained research personnel.

Eligible patients were invited to participate, and informed consent was obtained before inclusion. Briefly, exclusion criteria were inability to provide consent, valvular pathology warranting surgery, off-pump CABG, recent acute myocardial infarction, history of stroke or transient ischemic attack, structural brain abnormalities, carotid artery stenosis $>50 \%$, left ventricular ejection fraction $<30 \%$, atrial fibrillation, chronic renal disease (estimated glomerular filtration rate $<50 \mathrm{~mL} / \mathrm{min} / 1.73 \mathrm{~m}^{2}$ ), contraindications to MRI, upper extremity vascular pathology, and urgent operation. The predefined secondary exclusion criteria were newly diagnosed structural abnormalities on preoperative brain MRI, new-onset postoperative contraindications to MRI, and perioperative hemodynamic instability.

\section{Myocardial Revascularization}

Preoperatively, patients were given morphine and diazepam. For induction and maintenance of anesthesia we used midazolam, sufentanil, 
pancuronium bromide, and sevoflurane. Propofol was purposefully avoided, because of its alleged capacity to inhibit RIPC-inducing pathways. ${ }^{7,9}$ Surgical myocardial revascularization was performed with the use of cardiopulmonary bypass. A single period of aortic cross-clamping was used. A combination of antegrade and retrograde cardioplegia was used for myocardial protection.

\section{Study Intervention and Control Protocols}

RIPC was induced by 3 alternating cycles of upper extremity ischemia and reperfusion. Ischemic episodes were induced by inflating a blood pressure cuff to $200 \mathrm{~mm} \mathrm{Hg}$ for 5 minutes. The arm was then reperfused for 5 minutes by deflating the cuff, after which the sequence was repeated. In patients randomized to the sham procedure the blood pressure cuff was positioned, but without inducing alternating inflation and deflation cycles. The RIPC intervention protocol used in the present trial has been previously described and validated. ${ }^{7}$

\section{Structural Brain MRI}

We used a comprehensive array of imaging tools to assess discrete cerebral changes at the structural and functional levels. MRI was performed pre- and postoperatively on a $3 \mathrm{~T}$ magnetic resonance scanner (Magnetom TrioTim; Siemens, Erlangen, Germany) using a 32-channel head coil. Fluid attenuated inversion recovery sequences (repetition time [TR], $9000 \mathrm{~ms}$; echo time [TE], $87 \mathrm{~ms}$; voxel size, $0.9 \times 0.9 \times 4 \mathrm{~mm}$; field of view, $230 \times 183$; matrix, $204 \times 256$; flip angle, $130^{\circ}$ ) and diffusion-weighted imaging sequences (TR, $3100 \mathrm{~ms}$; TE, $92 \mathrm{~ms}$; voxel size, $1.8 \times 1.8 \times 5 \mathrm{~mm}$; field of view, $230 \times 230$; matrix, $128 \times 128$; baseline $1=0 \mathrm{~s} / \mathrm{mm}^{2}$; baseline $2,500 \mathrm{~s} / \mathrm{mm}^{2}$; baseline $3,1000 \mathrm{~s} / \mathrm{mm}^{2}$ ) were acquired and analyzed for this purpose. Detection of new ischemic brain lesions was on the basis of a comparison between pre- and postoperative MRI scans. New ischemic lesions were volumetrically quantified using Analyze 8.1 software (Mayo Clinic, Rochester, Minn).

\section{Resting-State Functional Connectivity MRI}

Resting-state functional connectivity MRI (rs-fcMRI) gradient echoplanar imaging sequences sensitive for blood oxygen level-dependent (BOLD) contrast were used. Functional images were coregistered with high-resolution magnetization-prepared rapid acquisition gradient-echo T1 images. Very stringent quality control of all BOLD images was performed across the investigated groups. Furthermore, all BOLD time series underwent high- $(0.009 \mathrm{~Hz})$ and low-pass $(0.08 \mathrm{~Hz})$ temporal filtering, nuisance signal removal from ventricles and deep white matter, global signal regression, 6 rigid-body motion correction parameters, and first derivatives using inhouse MATLAB tools (MATLAB R2016b; MathWorks, Natick, Mass). ${ }^{10}$ Structural and functional MRI data were preprocessed in line with the methods derived by the Human Connectome Project (HCP). ${ }^{11}$ The current state-of-the-art approach in spatial distortion correction, registration, and maximization of high-resolution signal-to-noise was implemented. ${ }^{12}$ An indepth depiction of the complex rs-fcMRI acquisition process, as well as specific quality control measures that were used are provided in the Appendix E1.

\section{Functional Connectivity Analyses}

Thalamic seeds were defined for each subject using automatic anatomical segmentation of high-resolution structural images. A "cortical ribbon" was defined along with corresponding subcortical voxels, which were combined to generate the Connectivity Informatics Technology Initiative (CIFTI) volume/surface "gray-ordinate" space. Pearson correlations were computed between the mean BOLD signal time series for the "associative" nuclei (used as 1 seed) and the BOLD time series at every other cortical and subcortical vertex in CIFTI gray-ordinate space. Specifically, we masked subjectspecific thalamus voxels with the thalamic nuclei projecting to the prefrontal, premotor, parietal, and temporal cortex, defined by the FMRIB Software Library (FSL) thalamus atlas. ${ }^{13}$ In doing so, we were able to test whether a thalamic nucleus with widespread associative-cortex connectivity would be affected by surgery and/or RIPC pretreatment. Functional connectivity data from 34 patients ( 14 from the RIPC group and 20 from the control group) met the quality control standards, and were included in subsequent analyses. Subjects were excluded for any of the following reasons: (1) poor structural scan, which yielded poor segmentation; (2) excessive head motion; or (3) poor signal quality of the BOLD scans.

\section{Neurocognitive Testing}

Montreal Cognitive Assessment (MOCA), Trail Making Test (TMT)-A and TMT-B were used for quantifying the neurocognitive status. Neurocognitive testing was performed preoperatively and on postoperative day 7 , mirroring the MRI imaging timeline. This strategy allowed for matching of neurocognitive test results with advanced brain imaging data. Cognitive dysfunction 7 days postoperatively has been reported to adversely affect quality of life, leading to premature abandonment of the labor market and financial dependency. ${ }^{14}$ Patients with cognitive decline early after surgery have been shown to be at risk for reduced cognitive functioning in the long term. ${ }^{15}$

Briefly, MOCA screens for discrete cognitive impairment, by evaluating distributed and focal cognitive domains, including attention, orientation, memory, executive function, conceptual thinking, and calculation. To negate the potential influence of a learning curve, patients were subjected to different iterations of the MOCA test pre- and postoperatively. Swiftness of cognitive processing, visual attentiveness, and executive functioning were evaluated with the bipartite TMT. Each of the 2 partitions of the TMT are represented by their own numerical outcome, and were analyzed separately. The neurocognitive protocol was described previously. ${ }^{10}$ Neurocognitive testing was performed by trained personnel blinded to the patient's allocation status.

\section{Primary and Secondary End Points}

The primary composite outcome was a binary end point of new brain ischemia on structural MRI and new impairment in neurocognition. The latter was defined as a decrease of minimally 1 standard deviation in 2 or more postoperative neurocognitive tests. Individual components of the primary outcome are presented in detail as secondary end points, rather than merely as a binary outcome. For new brain ischemic lesions the secondary end points included pooled ischemic volumes and overall number of lesions per patient. Postoperative neurocognitive dysfunction is presented for 99 paired neurocognitive tests performed in each group. The definition of abnormal neurocognitive test results mirrored the one used for the primary outcome (ie, a postoperative decrease of at least 1 standard deviation compared with the preoperative test result). Brain-wide associative thalamic resting-state functional connectivity profiles were acquired at 2 time points (pre- and postoperatively). The differences between pre- and postoperative functional connectivity profiles are presented as secondary trial end points. Major adverse cardiac and cerebrovascular events, defined as all-cause mortality, nonfatal myocardial infarction, stroke, and cardiovascular rehospitalization, were adjudicated at 3-month follow-up.

\section{Statistical Analysis}

Continuous data are presented as mean values \pm standard deviation or medians with interquartile ranges. The Shapiro-Wilk test was used to determine whether a continuous outcome was well modeled by a normal distribution. The $t$ test was used for analyzing normally distributed continuous variables, and the Mann-Whitney $U$ test was used for non-normally distributed variables.

Categorical variables and end points are presented as absolute numbers with percentages and were compared across groups using $2 \times 2$ contingency tables. Measures of association were derived from Fisher exact tests. The treatment effect is presented as odds ratios (ORs), with 2-sided $95 \%$ confidence intervals (CIs). A 2 -tailed $P$ value of $<.05$ was considered 
significant for all statistics. The data were processed using the IBM SPSS Statistics software package (version 20.0; Armonk, NY).

For the analysis of rs-fcMRI data, correlation maps were standardized for statistical analyses via Fisher r-to- $Z$ transformation. To test the Group $\times$ Time point interaction effect with the BOLD rs-fcMRI as the dependent measure, we computed a 2-way repeated measures mixed model analysis of variance with a factor of group (RIPC vs control group) and time point (pre-surgery vs post surgery). To achieve an appropriate level of whole-brain type I error protection, we applied nonparametric permutation testing using the FSL Permutation Analysis of Linear Models algorithm ${ }^{16}$ with 10,000 permutations. This approach circumvents the distributional assumptions (eg, normality) that might result in type I error inflation. ${ }^{17}$ Specifically, this approach provides a whole-brain type I error correction at the level of $P<.05$ across all voxels. In this way, results ensure that any surviving rs-fcMRI effect has to meet a highly conservative level of type I error control.

\section{RESULTS \\ Study Population}

A total of 1347 patients were operated on during the study period from March 2014 to December 2015 at our academic center. Of these 424 underwent isolated coronary artery bypass surgery, and entered the primary screening process. A total of 70 patients were randomized. Randomization was on the basis of computer-generated coding. Sealed envelopes containing the codes assured the implementation of the random allocation sequence. Patient enrollment and randomization are detailed in the Consolidated Standards of Reporting Trials flow diagram (Figures 1 and E1). The CONSORT 2010 checklist of information to include when reporting a randomized trial is provided in the Online Data Supplement.

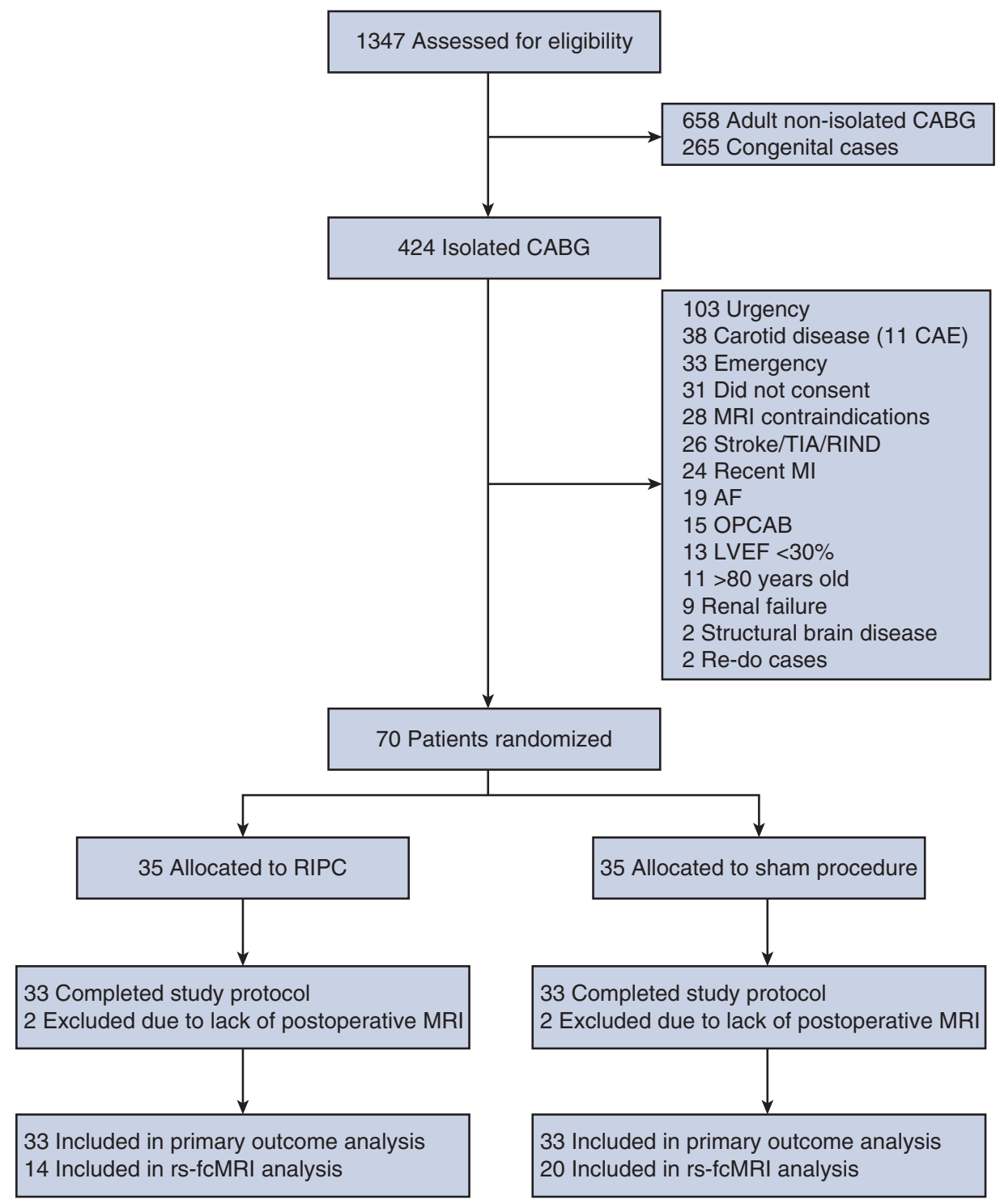

FIGURE 1. Consolidated Standards of Reporting Trials flow diagram. $C A B G$, Coronary artery bypass grafting; $C A E$, carotid endarterectomy; $M R I$, magnetic resonance imaging; TIA, transient ischemic attack; RIND, reversible ischemic neurological deficit; $M I$, myocardial infarction; $A F$, atrial fibrillation; $O P C A B$, off-pump coronary artery bypass; $L V E F$, left ventricular ejection fraction; $R I P C$, remote ischemic preconditioning; $r s-f c M R I$, resting-state functional connectivity magnetic resonance imaging. 
TABLE 1. Baseline demographic and clinical profiles of the study population $(N=66)$

\begin{tabular}{|c|c|c|c|}
\hline & Control group $(\mathbf{n}=33)$ & RIPC group $(\mathbf{n}=33)$ & $P$ \\
\hline \multicolumn{4}{|l|}{ Patient characteristics } \\
\hline Age, y & $62 \pm 8$ & $62 \pm 7$ & .736 \\
\hline Male sex, n (\%) & $26(79)$ & $28(85)$ & .751 \\
\hline Body mass index & $29 \pm 3$ & $29 \pm 4$ & 699 \\
\hline EuroSCORE II, \% & $1.03[0.85,1.49]$ & $1.14[0.86,1.73]$ & .590 \\
\hline Left ventricular ejection fraction, $\%$ & $57 \pm 10$ & $57 \pm 9$ & .927 \\
\hline Creatinine, $\mathrm{mmol} / \mathrm{L}$ & $82[70,103]$ & $90[74,99]$ & .586 \\
\hline Hyperlipidemia, n (\%) & $26(79)$ & $25(76)$ & 1.0 \\
\hline Diabetes mellitus, n (\%) & $14(42)$ & $10(30)$ & .443 \\
\hline Smoking history, n (\%) & $21(64)$ & $15(45)$ & .216 \\
\hline Hypertension, n (\%) & $27(82)$ & $29(88)$ & .733 \\
\hline Left main disease, n ( \%) & $8(24)$ & $5(15)$ & .537 \\
\hline Three-vessel disease, n (\%) & $24(73)$ & $26(79)$ & .775 \\
\hline \multicolumn{4}{|l|}{ Preoperative medications, $\mathrm{n}(\%)$} \\
\hline Clopidogrel & $8(24)$ & $11(33)$ & .587 \\
\hline Aspirin & $33(100)$ & $33(100)$ & 1.0 \\
\hline$\beta$-blocker & $29(88)$ & $31(94)$ & .672 \\
\hline ACE inhibitor & $25(76)$ & $26(79)$ & 1.0 \\
\hline Statin & $30(91)$ & $30(91)$ & 1.0 \\
\hline
\end{tabular}

RIPC, Remote ischemic preconditioning; $A C E$, angiotensin-converting enzyme.

RIPC was induced as per protocol in 35 of the 70 randomized patients. The remaining 35 patients underwent a sham procedure. Two patients in each group did not undergo postoperative MRI and were excluded because of protocol violation (2 because of pacemaker wires, 1 had a panic attack during imaging which was then aborted, and 1 withdrew consent). The remaining 66 patients completed the study protocol, and were included in further analyses. Rs-fcMRI images were coregistered with structural MRI on postoperative 7. Structural MRI provides the 3-dimensional anatomical template necessary for subsequent functional connectivity analyses. All 66 postoperative MRI scans ( 33 in each group) were analyzed for the presence of new ischemic lesions. Although 66 patients also underwent the rs-fcMRI protocol, not all of them were interpretable for assessment of brain-wide associative thalamic resting-state functional connectivity profiles because of strict quality control criteria.

The baseline demographic and clinical profiles as well as preoperative medication regimens were well balanced between the 2 groups (Table 1). Approximately three-quarters of patients had 3-vessel disease and approximately a fifth of the randomized patients had left main coronary artery disease, which is representative of the contemporary cardiac surgical practice. Standardized on-pump surgical myocardial revascularization was performed in all patients, with no demonstrable differences in operative data between the cohorts. An overview of the perioperative patient data is provided in Table 2.

\section{Primary End Point Results}

There was no between-group difference in the incidence of the composite primary end point (9 [27\%] in the RIPC group vs $8[24 \%]$ in the control group; OR, 1.17 [95\%
CI, 0.34-4.06]; $P=1.0$ ). The overall incidence of new ischemic brain lesions was not reduced by RIPC ( $24 \%$ [8/ 33] vs $21 \%$ [7/33], OR, 1.19 [95\% CI, 0.33-4.37]; $P=1.0)$. Two patients (6\%) in the RIPC group had at least 2 abnormal postoperative neurocognitive test results versus 4 patients $(12 \%)$ in the control group (OR, $0.47 ; 95 \% \mathrm{CI}$, $0.05-3.33 ; P=.672$ ).

\section{Volumetric Analysis of New Ischemic Brain Lesions}

Although the overall incidence of new ischemic brain lesions was not reduced by RIPC, the number of new ischemic lesions was higher in the control group. Seven

TABLE 2. Summary of perioperative data $(\mathbf{N}=66)$

\begin{tabular}{llll}
\hline & $\begin{array}{c}\text { Control } \\
\text { group } \\
(\mathbf{n}=\mathbf{3 3})\end{array}$ & $\begin{array}{c}\text { RIPC } \\
\text { group } \\
(\mathbf{n}=\mathbf{3 3})\end{array}$ & $\boldsymbol{P}$ \\
\hline Cross-clamp time, minutes & $57(39-71)$ & $54(40-68)$ & .989 \\
\hline CPB time, minutes & $70(53-90)$ & $78(62-93)$ & .441 \\
\hline Internal mammary use & $30(91)$ & $32(97)$ & .613 \\
\hline Number of grafts & $2(2-3)$ & $3(2-3)$ & .279 \\
\hline Postoperative AF & $9(27)$ & $8(24)$ & 1.0 \\
\hline Postoperative inotrope use & $8(24)$ & $8(24)$ & 1.0 \\
\hline Resternotomy for bleeding & $2(6)$ & $0(0)$ & .492 \\
\hline Renal replacement therapy & $0(0)$ & $1(1)$ & 1.0 \\
\hline Mechanical ventilation, hours & $6(5-8)$ & $7(6-9)$ & .069 \\
\hline 30-Day mortality & $0(0)$ & $0(0)$ & 1.0 \\
\hline MCS & $0(0)$ & $0(0)$ & 1.0 \\
\hline Data are pres
\end{tabular}

Data are presented as $\mathrm{n}(\%)$ or median (interquartile range). RIPC, Remote ischemic preconditioning; $C P B$, cardiopulmonary bypass; $A F$, atrial fibrillation; $M C S$, mechanical circulatory assistance. 
patients in the control group had 25 new ischemic lesions, whereas 8 patients in RIPC group had 11. The average number of lesions per patient was $3.6 \pm 3.5$ in the control group compared with $1.4 \pm 0.5$ lesions per patient in the RIPC group $(P=.152)$. The pooled volume of all new brain lesions was lower in the RIPC group compared with the control arm (157 [interquartile range, 125-231] vs 777 [interquartile range, 564-965] $\mathrm{mm}^{3} ; P=.004$ ). Neurocognitive, clinical, and structural brain imaging data are summarized in Table 3. Seventy-three percent of patients had brain ischemic volumes $<0.05 \%$ of total brain volume. ${ }^{18} \mathrm{We}$ observed a preponderance of new lesions in the right hemisphere (23/36 [64\%]). A detailed localization of new ischemic lesions is provided in Table E1.

\section{Effect of Surgical Procedure and RIPC on Associative Thalamic Connectivity}

We first characterized the main effect of surgical procedure (pre/post) and its effects on brain-wide associative thalamic rs-fcMRI for the entire studied population. Results showed that the resting-state functional connectivity profiles for the associative thalamus are markedly reduced across a wide range of brain structures after surgery (Figure 2). For completeness the results are shown for thresholded and threshold-free analyses, to allow for full qualitative inspection of the effects. This effect was highly robust and survived brain-wide type I error protection, indicating reductions of brain-wide associative thalamic connectivity for the RIPC $(t=3.31 ; P<.01)$ and the control $(t=3.52 ; P<.01)$ groups (Figure 3$)$. Figures 3 and 4 illustrate post hoc pairwise type I error protected effects of surgery for each of the groups. Although some qualitative differences emerged, these effects were markedly similar across the RIPC and control groups. In line with this observation, there were no significant differences between the groups in rs-fcMRI either pre- or post surgery. This was also reflected by an absence of a significant Group $\times$ Time point interaction. Collectively, these effects clearly establish distinct reductions of brain-wide connectivity profiles after surgery for associative thalamic nuclei, which are critical for executive and cognitive function. These effects were not influenced by upper limb RIPC.

\section{Neurocognitive Testing}

All patients were subjected to neuropsychological testing pre- and postoperatively (MOCA, TMT-A, and TMT-B). This yielded a total of 99 comparisons between preoperative and postoperative values in each group, and allowed for analysis of trends (Table 3). Meeting our prespecified criteria we found that 16 of $99(16 \%)$ tests were abnormal in the control cohort versus 7 of $99(7 \%)$ in the RIPC group (OR, 0.40; 95\% CI, 0.14-1.09; $P=.074$ ). Five patients $(15 \%)$ in the RIPC group had at least 1 abnormal neurocognitive test result compared with 11 patients $(33 \%)$ in the control group (OR, 0.36; 95\% CI, 0.09-1.35; $P=.15$ ). Of note, 5 patients in the control cohort were unable to complete the postoperative TMT-B test compared with 1 patient in the RIPC arm (5/33 [15\%] vs $1 / 33$ [3\%]; $P=.197)$, despite the fact that subjects were given an additional 100 seconds to the original test design for completion. The statistically nonsignificant numerical decrease in the incidence of periprocedural delirium mirrored the aforementioned neurocognitive results $(4[12 \%]$ in the intervention arm vs $6[18 \%]$ in the control arm; OR, $0.62 ; 95 \% \mathrm{CI}$, $0.13-2.88 ; P=.733$ ).

\section{Periprocedural Results}

Patients in the intervention arm had similar durations of mechanical ventilator support (7 [interquartile range, 6-9] vs 6 [interquartile range, 5-8] hours; $P=.069$ ). We found no imbalances in the incidence of common perioperative complications, such as re-exploration for bleeding, perioperative atrial fibrillation, and acute kidney injury (Table 2).

TABLE 3. Neurocognitive, clinical, and brain imaging data $(n=66)$

\begin{tabular}{|c|c|c|c|c|}
\hline Outcome measure & Control group $(\mathbf{n}=\mathbf{3 3})$ & RIPC group $(\mathbf{n}=33)$ & Odds ratio $(95 \% \mathrm{CI})$ & $\boldsymbol{P}$ \\
\hline Primary composite end point & $8(24)$ & $9(27)$ & $1.17(0.34-4.06)$ & 1.0 \\
\hline New ischemic lesions & $7(21)$ & $8(24)$ & $1.19(0.33-4.37)$ & 1.0 \\
\hline Patients with $\geq 2$ abnormal NCTs & $4(12)$ & $2(6)$ & $0.47(0.05-3.33)$ & .672 \\
\hline Patients with 1 abnormal NCT & $11(33)$ & $5(15)$ & $0.36(0.09-1.35)$ & .150 \\
\hline Total abnormal NCTs & $16(16)$ & $7(7)$ & $0.40(0.14-1.09)$ & .074 \\
\hline New ischemic brain lesions & $3.6 \pm 3.5$ & $1.4 \pm 0.5$ & N/A & .152 \\
\hline New brain ischemic volume, $\mathrm{mm}^{3}$ & $777(564-965)$ & $157(125-231)$ & N/A & .004 \\
\hline TIA & $1(3)$ & $0(0)$ & N/A & 1.0 \\
\hline Stroke & $0(0)$ & $0(0)$ & N/A & 1.0 \\
\hline Delirium & $6(18)$ & $4(12)$ & $0.62(0.13-2.88)$ & .733 \\
\hline
\end{tabular}

Data are presented as n (\%), median (interquartile range), or mean \pm standard deviation, except where otherwise noted. RIPC, Remote ischemic preconditioning; $C I$, confidence interval; NCT, neurocognitive test; N/A, not applicable; TIA, transient ischemic attack. 

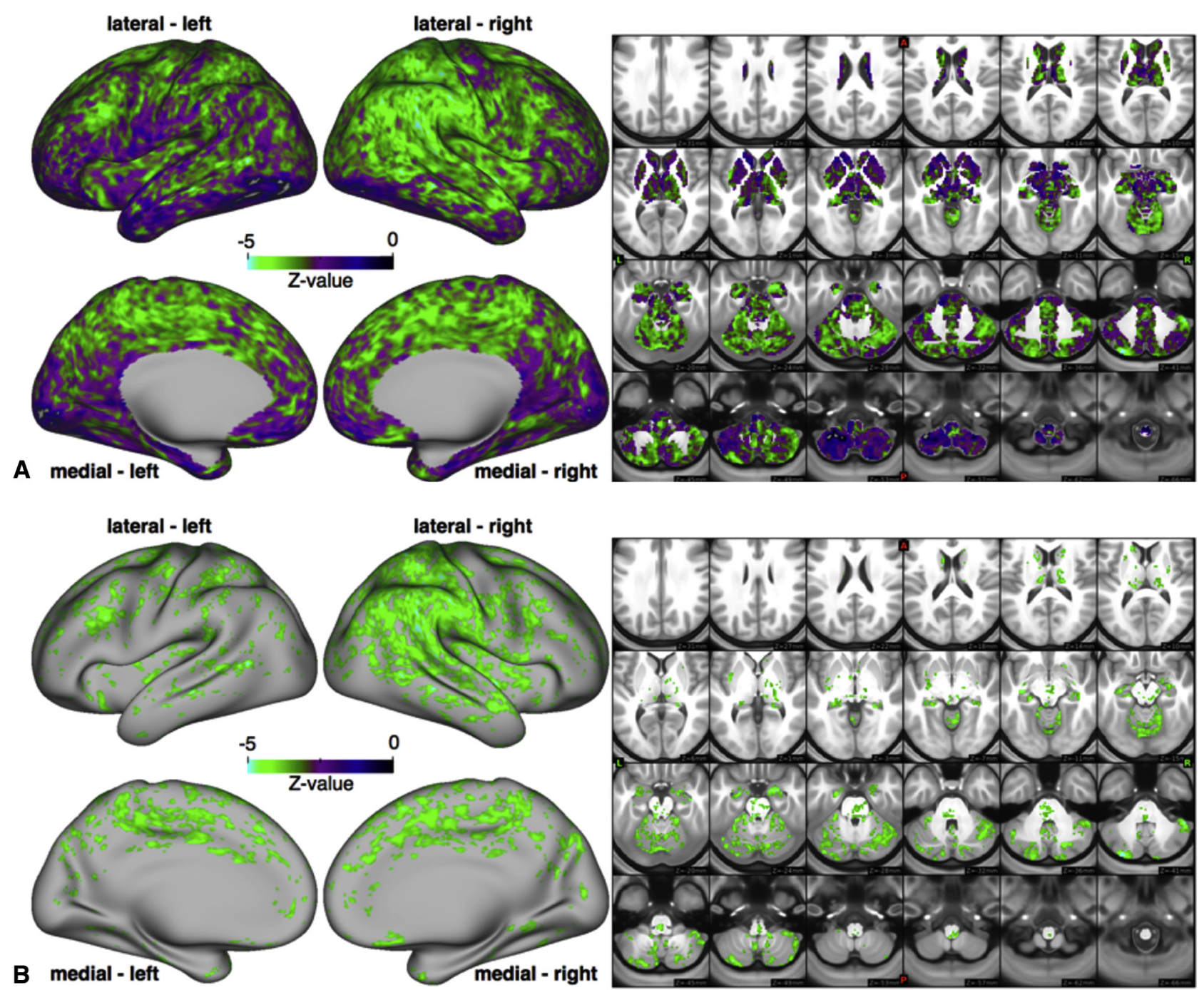

FIGURE 2. Functional connectivity profiles on resting-state magnetic resonance imaging. A, Surface and volume maps showing the threshold-free dependent-samples $t$ test for pre/post surgical effects on brain-wide associative thalamic resting-state functional connectivity for the entire study population. B, Whole-brain protected effect of surgical procedure for the associative thalamic nucleus. Contrast (threshold $\mathrm{Z}$ value $>3.5$ ) on surface and volume maps indicates brain-wide adverse effects of surgery on associative thalamic resting-state functional connectivity. All regions shown in green indicate reduced connectivity after surgery, expressed as a $\mathrm{Z}$ value and denoted by the color bar.

We could not show any benefit of RIPC on the release of troponin $\mathrm{T}$ on postoperative day $1(0.45 \mathrm{ng} / \mathrm{mL}[0.30-0.75$ $\mathrm{ng} / \mathrm{mL}]$ in the control arm vs $0.47 \mathrm{ng} / \mathrm{mL}[0.30-0.70 \mathrm{ng} /$ $\mathrm{mL}]$ in the RIPC arm; $P=.734$ ), or the use of periprocedural inotropic support ( $8[24 \%]$ vs $8[24 \%] ; P=1.0$ ). Postoperative peak creatinine values were similar between the investigated and control cohorts $(90 \mathrm{mmol} / \mathrm{L}$ [74-99 $\mathrm{mmol} / \mathrm{L}]$ vs $82 \mathrm{mmol} / \mathrm{L}$ [70-103 $\mathrm{mmol} / \mathrm{L}]$, respectively; $P=.822$ ). The incidence of acute kidney injury on the basis of RIFLE (Risk, Injury, Failure, Loss, End-stage renal failure) criteria was $12 \%(4 / 33)$ in the RIPC group, and $9 \%(3 / 33)$ in the control group, without demonstrable intergroup variability $(P=1.0)$. One patient in the RIPC group required renal replacement therapy. None of the patients in either cohort required mechanical circulatory assistance and there was no 30-day mortality.

The incidence of major adverse cardiac and cerebrovascular events was $3 \%(1 / 33)$ in the RIPC group, and $6 \%$ $(2 / 33)$ in the control group $(P=1.0)$. All events were driven by cardiovascular hospitalizations. The patient in the RIPC group had peripheral vascular ischemia, whereas both patients in the control group were managed for congestive heart failure.

\section{DISCUSSION}

Alternating episodes of sublethal ischemia and reperfusion in nonvital tissues mobilize the endogenous potential for mitigating injury precipitated by a prolonged ischemic 


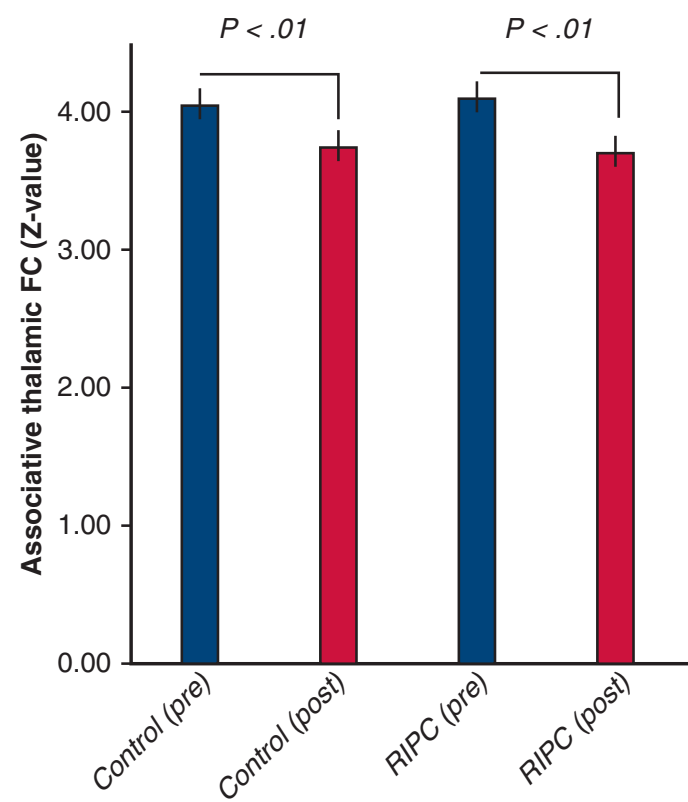

FIGURE 3. Functional connectivity effects of the surgical procedure for the associative thalamic nucleus extracted across all regions. Results indicate robust effects (expressed as $\mathrm{Z}$ values) for the remote ischemic preconditioning (RIPC); $t=3.31 ; P<.01]$ and the control groups $(t=3.52$; $P<.01)$. No significant intergroup interactions emerged. The black error bars indicate the standard error of the mean. $F C$, Functional connectivity.

event in vital organs. ${ }^{19}$ In this single-center prospective randomized trial we found that RIPC did not reduce the overall incidence of new ischemic brain lesions, but it did significantly reduce their absolute numbers and their total volume. There are 2 major zones of injury within an ischemic brain lesion: the core ischemic zone and the ischemic penumbra. The latter denotes an ischemic territory that harbors potentially salvageable cerebral tissue. Endogenous and exogenous protection mechanisms are more likely to be effective within the ischemic penumbra than the core ischemic zone. This might potentially explain why RIPC was efficient in reducing the size of the ischemic lesion, but failed to reduce the overall incidence on new ischemic lesions.

Controversy has surrounded the efficacy of RIPC in improving ischemic resistance in cardiac surgical patients. Meybohm and colleagues reported that upper-limb RIPC was unsuccessful in reducing a composite end point of death, myocardial infarction, stroke, or acute renal failure. ${ }^{8}$ Contrariwise, RIPC provided perioperative myocardial protection in the studies by Thielmann and colleagues ${ }^{7}$ and Venugopal and colleagues. ${ }^{20}$ A potentially important difference between the trials was that in the study by Meybohm and colleagues, a propofol-based anesthetic regimen was used universally in all patients, ${ }^{8}$ whereas in the latter 2 trials propofol was used sparingly. ${ }^{7,20}$ Taking into account that propofol might interfere with cardioprotective signaling upstream of signal transducer and activator of transcription 5 , this disparity in trial design might have hindered the efficacy of RIPC in the study that routinely used propofol. ${ }^{7,8,20,21}$ We avoided the use of propofol in the present trial, to dismiss it as a confounder.

The overall incidence of clinically indiscernible ischemic lesions on brain MRI among patients who underwent elective CABG in our study was $23 \%$. This incidence was lower than the $31 \%$ observed by Patel and colleagues, likely because of the fact that their study included a more heterogeneous patient population with a higher embolic risk burden. ${ }^{22}$ Additionally, patients with preoperative brain ischemic lesions have an increased risk of new postoperative brain ischemia ${ }^{22}$ and were, for this reason, selectively excluded from our trial. The normal brain volume ranges between 1.130 .000 to $1.260 .000 \mathrm{~mm}^{3}$. The pooled ischemic volumes observed in our patient groups seem very discrete in comparison. ${ }^{23}$ Volumetric quantification of new ischemic lesions in our trial showed that they were predominantly smaller than $0.05 \%$ of the total brain volume. ${ }^{18}$ The clinical relevance of these new ischemic brain lesions remains unknown, and might be a substrate for future studies. In contrast to the study by Patel and colleagues, ${ }^{22}$ we documented a larger proportion of lesions in the right hemisphere.

We observed a numerical reduction in neurocognitive performance in the control group compared with the RIPC arm, which remained below the threshold of statistical significance. Also of note, we found that the preoperative TMT-A and TMT-B test results in both of our cohorts were numerically lower compared with the normative control data of healthy subjects within the same age group. ${ }^{24}$ The optimal timing of neurocognitive outcome testing after cardiac surgery is subject to debate. We opted to chronologically match neurocognitive testing with MRI imaging to obtain a multifaceted impression of postoperative brain changes.

One of the most unique aspects of our study was the quantifiable appraisal of brain-wide connectivity profiles for associative thalamic nuclei after exposure to cardiac surgical trauma. We documented pronounced reductions in brain-wide connectivity profiles in regions known to be critical for executive functioning and other cognitive subdomains. These have been previously described as impaired after cardiac surgery. ${ }^{6}$ Our study was not designed to assess whether the registered changes in resting-state functional brain connectivity are only transient in nature or portend long-term sequelae.

The etiology of the reduction in neurocognitive performance seen after CABG remains elusive. We hypothesize that the reduction in brain-wide associative thalamic functional connectivity, coupled with new ischemic lesions on structural brain MRI, provide an explanation for clinically discrete but relevant alterations in neurocognitive performance. Our data corroborate the link between alterations 

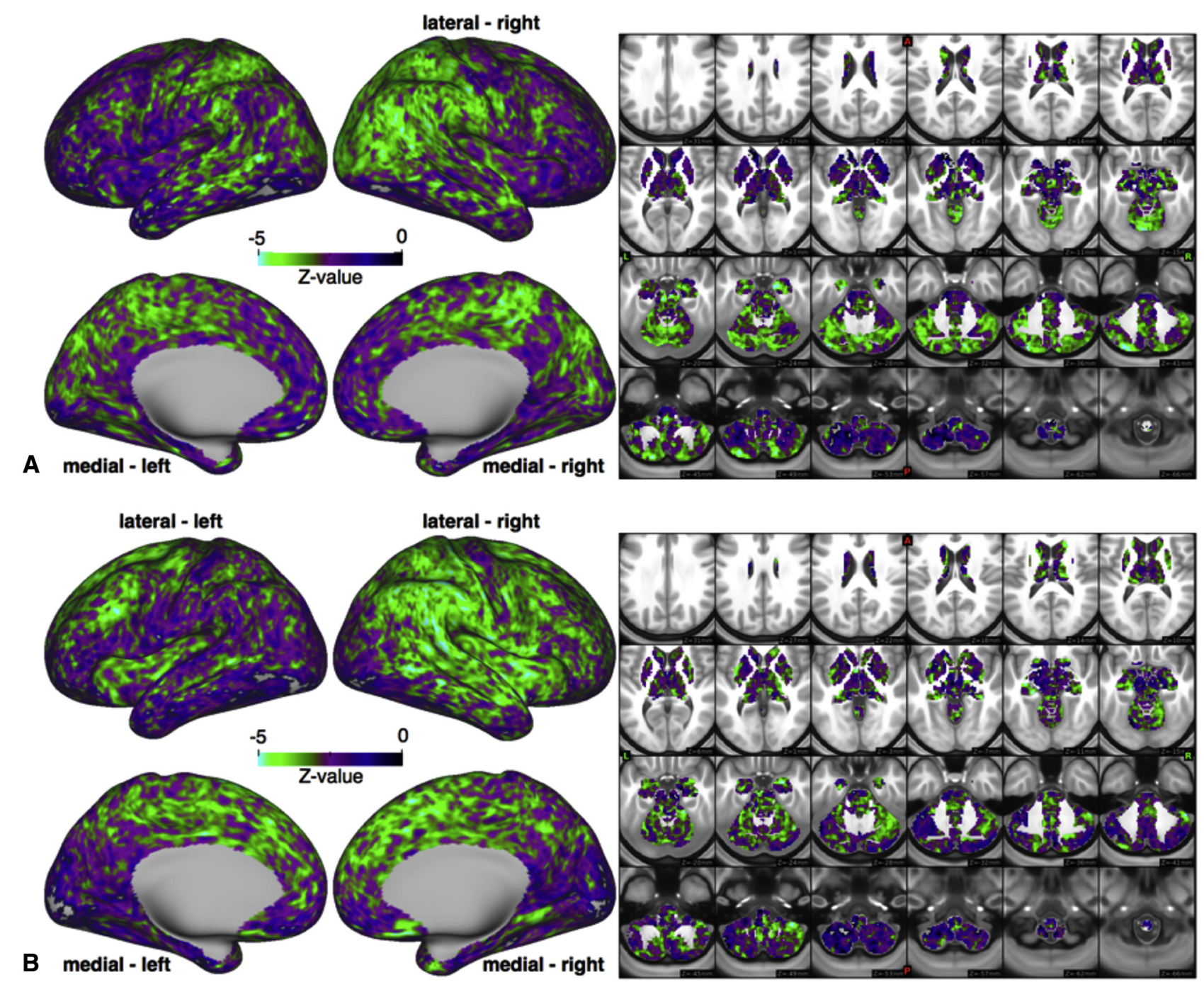

B medial - left

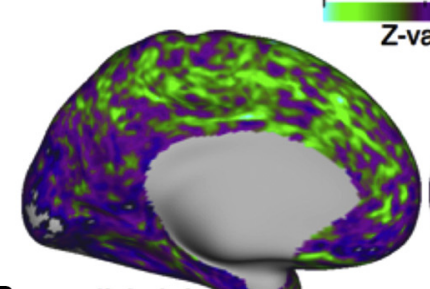

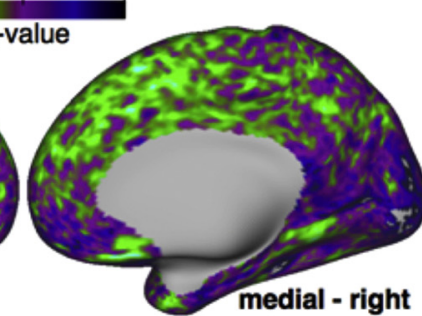

FIGURE 4. Effects of surgical procedure on brain-wide associative thalamic resting-state functional connectivity. A, Surface and volume maps showing the threshold-free dependent-samples $t$ test for pre/post surgical effects in the control group. B, Surface and volume maps showing the threshold-free dependent-samples $t$ test for pre/post surgical effects on brain-wide associative thalamic resting-state functional connectivity for the group receiving remote ischemic preconditioning pretreatment. All regions shown in green indicate reduced connectivity after surgery, expressed as a $\mathrm{Z}$ value.

in resting-state functional brain connectivity and postoperative cognitive dysfunction described recently in a small noninterventional study. ${ }^{25}$

Our results did not reveal a Group $\times$ Time point interaction in this analysis. However, these effects indicate that using rs-fcMRI as a marker of surgical effects on the central nervous system can capture robust and measurable changes.

We found no effect of RIPC on troponin release in our study. Similarly, we found no evidence that RIPC provided a renoprotective effect. Our results did not corroborate the higher incidence of perioperative arrhythmias induced by RIPC observed in a previous trial. ${ }^{26}$

One of the major strengths of our study was the adherence to a very comprehensive brain imaging protocol, which combined structural and functional imaging sequences. Furthermore, we completely eliminated propofol from our anesthetic protocol, because of its inhibitory effect on ischemic preconditioning pathways. ${ }^{21}$

A graphical summary of our trial is presented in Figure 5. The tripartite figure succinctly encapsulates the intervention, effector response, and data analysis in individual panels.

\section{Limitations}

We acknowledge several limitations relevant to our study. Our study was negative with the respect to the primary outcome. The incidence of the primary outcome in our studied control group was $24 \%$. To show a $20 \%$ reduction in the primary outcome with a power of 0.90 , we would need to enroll $>1400$ patients in each arm to reject the null 


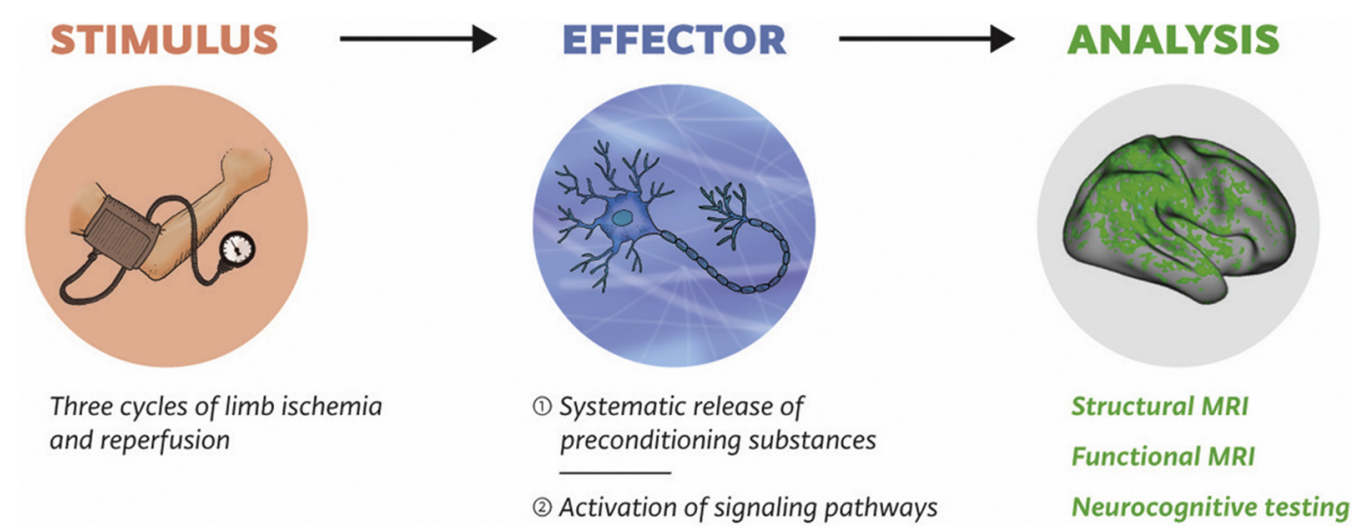

FIGURE 5. Impact of Remote Ischemic Preconditioning Preceding Coronary Artery Bypass Grafting on Inducing nEuroprotection (RIPCAGE) graphical abstract. Remote ischemic preconditioning was induced by alternating periods of arm ischemia and reperfusion (stimulus). The subsequent panel illustrates the effector response, followed by imaging data analysis. The final panel captures the brain-wide postoperative reductions in associative thalamic functional connectivity (shown in green). MRI, Magnetic resonance imaging.

hypothesis. ${ }^{27}$ In our study we used advanced MRI imaging (brain-wide associative thalamic resting-state functional connectivity), which would make the challenge of such enrollment prohibitive. Therefore, our study should be considered a hypothesis-testing work. The limited sample size might have also resulted in underestimation of the importance of potential risk factors that might have predisposed the brain to perioperative MRI-detectable injury. However, although small according to conventional standards, our trial is the largest prospective randomized study, to our knowledge, in the cardiac surgical patient population on the effects of RIPC on the brain using advanced MRI neuroimaging. Our study was not powered to test either the effect of RIPC on postoperative kidney function nor troponin release. The absence of any effect of RIPC on

Functional connectivity profiles on rs-fcMRI (4)

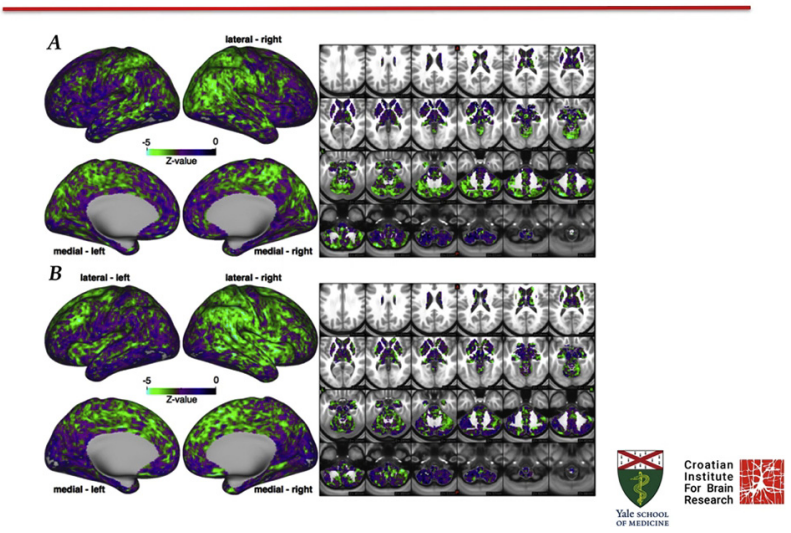

VIDEO 1. The effect of RIPC on the incidence and volume of new brain ischemic lesions after CABG; resting-state functional connectivity and neurocognitive impairment after $\mathrm{CABG}$, and their relationship with preoperative RIPC. Video available at: https://www.jtcvs.org/article/S00225223(18)32516-9/fulltext. postoperative troponin release or kidney function seen in our study should, therefore, be considered with this caveat.

Another limitation of the present trial is the absence of routine epiaortic ultrasound scanning in all of the study participants. All patients received a comprehensive intraoperative transesophageal echocardiographic evaluation, which also focused on potential ascending aortic pathology. This was coupled with manual aortic palpation to identify potential aortic plaque. Patients in whom a high index of suspicion for aortic plaque was detected would have undergone additional epiaortic ultrasound scanning. Significant aortic atheroma would have prompted an intraoperative alteration in cannulation and clamping strategies or a transition to offpump CABG. In both cases these study participants would have been automatically excluded from the present trial. Because our study population, however, came from a lowrisk stratum of CABG patients, none of them were found to have aortic atheroma that would warrant modification to our standardized technique of on-pump CABG.

RIPC leads to a release of multiple active substances, which lead to activation of downstream signaling pathways. $^{28}$ The challenge remains to identify the best set of biomarkers to measure the response to RIPC. In our study we did not assay potential biomarkers of RIPC. It is, therefore, impossible to ascertain from our data whether the applied preconditioning protocol was neutral to the primary outcome because of failure of RIPC to reduce the incidence of the primary end point, or whether the applied protocol failed to induce a preconditioning response.

Finally, the strict quality control criteria that we imposed on our rs-fcMRI data rendered some of the acquired rsfcMRI studies inadequate. Of the total number of rsfcMRI that were acquired we found 14 from the RIPC group and 20 from the control group to be interpretable after being subjected to our quality control filter. The between-group analysis on the basis of rs-fcMRI data cannot, therefore, 
be considered to be derived from a randomized study design.

\section{CONCLUSIONS}

RIPC did not reduce the incidence of the primary outcome nor did it protect against the postoperative reduction in thalamic functional connectivity profiles. However, upperextremity RIPC was effective in reducing the pooled volume of new ischemic brain lesions (Video 1). The results of the present trial might promote the conduct of a future, larger scale study designed to evaluate the utility of RIPC as an avenue of neuroprotection in the cardiac surgical arena.

\section{Conflict of Interest Statement}

Dr Anticevic disclosed a financial relationship with BlackThorn Thereaputics (San Francisco, Calif). All other authors have nothing to disclose with regard to commercial support.

\section{References}

1. McDonagh DL, Berger M, Mathew JP, Graffagnino C, Milano CA, Newman MF Neurological complications of cardiac surgery. Lancet Neurol. 2014;13:490-502.

2. Duan X, Zhu T, Chen C, Zhang G, Zhang J, Wang L, et al. Serum glial cell linederived neurotrophic factor levels and postoperative cognitive dysfunction after surgery for rheumatic heart disease. J Thorac Cardiovasc Surg. 2018;155: 958-65.e1.

3. Nguyen Q, Uminski K, Hiebert BM, Tangri N, Arora RC. Midterm outcomes after postoperative delirium on cognition and mood in patients after cardiac surgery. J Thorac Cardiovasc Surg. 2018;155:660-7.e2.

4. Pilcher JM, Young P, Weatherall M, Rahman I, Bonser RS, Beasley RW. A systematic review and meta-analysis of the cardioprotective effects of remote ischaemic preconditioning in open cardiac surgery. J R Soc Med. 2012;105:436-45.

5. Narayanan SV, Dave KR, Perez-Pinzon MA. Ischemic preconditioning and clinical scenarios. Curr Opin Neurol. 2013;26:1-7.

6. Gasparovic H, Borojevic M, Malojcic B, Gasparovic K, Biocina B. Single aortic clamping in coronary artery bypass surgery reduces cerebral embolism and improves neurocognitive outcomes. Vasc Med. 2013;18:275-81.

7. Thielmann M, Kottenberg E, Kleinbongard P, Wendt D, Gedik N, Pasa S, et al. Cardioprotective and prognostic effects of remote ischaemic preconditioning in patients undergoing coronary artery bypass surgery: a single-centre randomised, double-blind, controlled trial. Lancet. 2013;382:597-604.

8. Meybohm P, Bein B, Brosteanu O, Cremer J, Gruenewald M, Stoppe C, et al. A multicenter trial of remote ischemic preconditioning for heart surgery. $N$ Engl J Med. 2015:373:1397-407.

9. Hausenloy DJ, Candilio L, Evans R, Ariti C, Jenkins DP, Kolvekar S, et al, Remote ischemic preconditioning and outcomes of cardiac surgery. $N$ Engl $J$ Med. 2015;373:1408-17.

10. Gasparovic H, Kopjar T, Rados M, Anticevic A, Rados M, Malojcic B, et al. Impact of remote ischemic preconditioning preceding coronary artery bypass grafting on inducing neuroprotection (RIPCAGE): study protocol for a randomized controlled trial. Trials. 2014;15:414.
11. Glasser MF, Sotiropoulos SN, Wilson JA, Coalson TS, Fischl B, Andersson JL, et al. The minimal preprocessing pipelines for the Human Connectome Project. Neuroimage. 2013;80:105-24.

12. Glasser MF, Coalson TS, Robinson EC, Hacker CD, Harwell J, Yacoub E, et al. A multi-modal parcellation of human cerebral cortex. Nature. 2016;536:171-8.

13. Behrens TE, Johansen-Berg H, Woolrich MW, Smith SM, WheelerKingshott CA, Boulby PA, et al. Non-invasive mapping of connections between human thalamus and cortex using diffusion imaging. Nat Neurosci. 2003;6: 750-7.

14. Steinmetz J, Christensen KB, Lund T, Lohse N, Rasmussen LS, ISPOCD Group. Long-term consequences of postoperative cognitive dysfunction. Anesthesiology. 2009; 110:548-55.

15. Newman MF, Kirchner JL, Phillips-Bute B, Gaver V, Grocott H, Jones RH, et al. Longitudinal assessment of neurocognitive function after coronary-artery bypass surgery. N Engl J Med. 2001;344:395-402.

16. Winkler AM, Ridgway GR, Webster MA, Smith SM, Nichols TE. Permutation inference for the general linear model. Neuroimage. 2014;92:381-97.

17. Eklund A, Nichols TE, Knutsson H. Cluster failure: why fMRI inferences for spatial extent have inflated false-positive rates. Proc Natl Acad Sci U S A. 2016;113:7900-5.

18. Luders E, Steinmetz H, Jäncke L. Brain size and grey matter volume in the healthy human brain. Neuroreport. 2002;13:2371-4.

19. Hausenloy DJ, Yellon DM. Remote ischaemic preconditioning: underlying mechanisms and clinical application. Cardiovasc Res. 2008;79:377-86.

20. Venugopal V, Hausenloy DJ, Ludman A, Di Salvo C, Kolvekar S, Yap J, et al. Remote ischaemic preconditioning reduces myocardial injury in patients undergoing cardiac surgery with cold-blood cardioplegia: a randomised controlled trial. Heart. 2009;95:1567-71.

21. Kottenberg E, Musiolik J, Thielmann M, Jakob H, Peters J, Heusch G. Interference of propofol with signal transducer and activator of transcription 5 activation and cardioprotection by remote ischemic preconditioning during coronary artery bypass grafting. J Thorac Cardiovasc Surg. 2014;147:376-82.

22. Patel N, Horsfield MA, Banahan C, Janus J, Masters K, Morlese J, et al. Impact of perioperative infarcts after cardiac surgery. Stroke. 2015;46:680-6.

23. Cosgrove KP, Mazure CM, Staley JK. Evolving knowledge of sex differences in brain structure, function, and chemistry. Biol Psychiatry. 2007;62:847-55.

24. Ashendorf L, Jefferson AL, O'Connor MK, Chaisson C, Green RC, Stern RA. Trail Making Test errors in normal aging, mild cognitive impairment, and dementia. Arch Clin Neuropsychol. 2008;28:129-37.

25. Browndyke JN, Berger M, Harshbarger TB, Smith PJ, White W, Bisanar TL, et al. Resting-state functional connectivity and cognition after major cardiac surgery in older adults without preoperative cognitive impairment: preliminary findings. J Am Geriatr Soc. 2017;65:e6-12.

26. Lucchinetti E, Bestmann L, Feng J, Freidank H, Clanachan AS, Finegan BA, et al. Remote ischemic preconditioning applied during isoflurane inhalation provides no benefit to the myocardium of patients undergoing on-pump coronary artery bypass graft surgery: lack of synergy or evidence of antagonism in cardioprotection? Anesthesiology. 2012;116:296-310.

27. Faul F, Erdfelder E, Lang AG, Buchner A. G*Power 3: a flexible statistical power analysis program for the social, behavioral, and biomedical sciences. Behav Res Methods. 2007;39:175.e191.

28. Koch S, Della-Morte D, Dave KR, Sacco RL, Perez-Pinzon MA. Biomarkers for ischemic preconditioning: finding the responders. J Cereb Blood Flow Metab. 2014;34:933-41.

Key Words: remote ischemic preconditioning, CABG, MRI, functional connectivity, neurocognitive outcome 


\section{APPENDIX E1. METHODS Rs-fcMRI}

Rs-fcMRI gradient echo-planar imaging sequences sensitive for BOLD contrast were used (TR, $3000 \mathrm{~ms}$; TE, $31 \mathrm{~ms}$; voxel size, $3.4 \times 3.4 \times 3 \mathrm{~mm}$; field of view, $220 \times 220$; matrix, $64 \times 64$; flip angle, $90^{\circ}$ ) for a period of 10 minutes and 8 seconds. Functional images were coregistered with highresolution magnetization-prepared rapid acquisition gradient-echo T1 images (TR, $1900 \mathrm{~ms}$; TE, $2.52 \mathrm{~ms}$; voxel size, $1 \times 1 \times 1 \mathrm{~mm}$; field of view, $250 \times 250$; matrix, $246 \times 256$, flip angle, $9^{\circ}$ ).

Quality control of all BOLD images across the investigated groups included: (1) signal-to-noise ratios $>100$, computed by obtaining the mean signal and standard deviation for a given slice across the BOLD run, while excluding all non-brain voxels across all frames ${ }^{\mathrm{E}}$; (2) no BOLD run with a single frame movement greater than 1 functional voxel; and (3) movement scrubbing. ${ }^{\text {E1,E2 }}$

\section{Data Preprocessing}

MRI data were preprocessed in line with the HCP, and described in detail elsewhere. ${ }^{\text {E3 }}$ These open-source HCP algorithms were further optimized for compatibility with legacy single-band data in this study. All processing methods closely followed the minimal processing pipelines as outlined previously, ${ }^{\mathrm{E} 3}$ with a few key modifications. The adapted HCP pipeline included the following steps: (1) the T1-weighted images were corrected for bias-field distortions and warped to the standard Montreal Neurological Institute-152 brain template through a combination of linear and nonlinear transformations using the FSL linear image registration tool and nonlinear image registration tool ${ }^{\mathrm{E} 4}$; (2) the FreeSurfer (Harvard University, Cambridge, Mass) recon-all pipeline was used to compute brainwide segmentation of gray and white matter to produce individual cortical and subcortical anatomical segmentation ${ }^{\mathrm{E} 5}$; (3) cortical surface models were generated for pial and white matter boundaries as well as segmentation masks for each subcortical gray matter voxel. Using the pial and white matter surface boundaries, a "cortical ribbon" was defined along with corresponding subcortical voxels, which were combined to generate the CIFTI volume/surface "gray-ordinate" space for each individual subject, which drastically reduces file management for combined surface and volume analyses and visualization and establishes a combined cortical surface and subcortical volume coordinate system ${ }^{\mathrm{E} 3}$; (4) the cortical surfaces were then registered to the group average HCP atlas using surface-based registration on the basis of cortical landmark features, whereas the subcortical "volume" component of the image was brought into group atlas alignment via nonlinear registration. ${ }^{\text {E3 }}$ The BOLD data were motion-corrected and aligned to the middle frame of every run using the FSL linear image registration tool. In turn, a liberal brain mask was applied to exclude signal from nonbrain tissue. After initial processing in Neuroimaging Informatics Technology Initiative volume space, BOLD data were converted to the CIFTI gray matter matrix using sampling from the anatomically-defined gray matter cortical ribbon whereas the subcortical voxels were isolated using subject-specific FreeSurfer segmentation. The subcortical volume component of the BOLD data were then aligned to the group atlas as part of the Neuroimaging Informatics Technology Initiative processing in a single transform step that concatenates all of the transform matrixes for each previous processing step (ie, motion correction, registration, distortion correction). This produced a single nonlinear transformation to minimize interpolation cost. In turn, the cortical surface component of the CIFTI file was aligned to the HCP atlas using surface-based nonlinear deformation on the basis of sulcal features. After these HCP preprocessing steps, a high-pass filter $(>0.5 \mathrm{~Hz})$ was applied to the BOLD time series to remove low temporal frequencies and scanner drift. In-house MATLAB tools were then used to compute the signal in the ventricles, deep white matter, and across all gray matter voxels (proxy of global mean signal regression to address spatially pervasive sources of artifacts). ${ }^{\mathrm{E} 6}$ These time series were modeled as nuisance variables and were regressed out of the gray matter voxels. In subsequent analyses we used the residual BOLD time series after these denoising steps.

\section{Functional Connectivity Analyses}

Thalamic seeds were first defined individually for each subject using automatic anatomical segmentation of high-resolution structural images via FreeSurfer software as part of the HCP minimal preprocessing pipelines. These structures were then used as "seeds," as conducted in our previous work. ${ }^{\mathrm{E} 7}$ Several analyses were performed to address confounds potentially introduced during data acquisition or processing. For BOLD images, frames with significant head movement were flagged on the basis of algorithms and intensity thresholds recommended for multiband data. ${ }^{\mathrm{E}}$ Temporal signal-to-noise ratio was calculated for each subject as the ratio of mean BOLD signal to its standard deviation over time. Movement (percentage of flagged frames) and signal-to-noise correlations with rs-fcMRI effects were examined for both groups. Global signal regression was included as a preprocessing step for the main analyses, but functional connectivity was also recomputed for the data without global signal regression to ensure that the effects were comparable at the whole-brain level, and within the specific regions of interest derived from permutation testing.

\section{E-References}

E1. Power JD, Barnes KA, Snyder AZ, Schlaggar BL, Petersen SE. Spurious but systematic correlations in functional connectivity MRI networks arise from subject motion. Neuroimage. 2012;59:2142-54.

E2. Power JD, Barnes KA, Snyder AZ, Schlaggar BL, Petersen SE. Steps toward optimizing motion artifact removal in functional connectivity MRI; a reply to Carp. Neuroimage. 2013;76:439-41.

E3. Glasser MF, Sotiropoulos SN, Wilson JA, Coalson TS, Fischl B, Andersson JL, et al. The minimal preprocessing pipelines for the Human Connectome Project. Neuroimage. 2013;80:105-24.

E4. Jenkinson M, Bannister P, Brady M, Smith S. Improved optimization for the robust and accurate linear registration and motion correction of brain images. Neuroimage. 2002;17:825-41.

E5. Reuter M, Schmansky NJ, Rosas HD, Fischl B. Within-subject template estimation for unbiased longitudinal image analysis. Neuroimage. 2012;61: 1402-18.

E6. Power JD, Plitt M, Laumann TO, Martin A. Sources and implications of whole-brain fMRI signals in humans. Neuroimage. 2017; 146:609-25.

E7. Anticevic A, Cole MW, Repovs G, Murray JD, Brumbaugh MS, Winkler AM et al. Characterizing thalamo-cortical disturbances in schizophrenia and bipolar illness. Cereb Cortex. 2014;24:3116-30. 


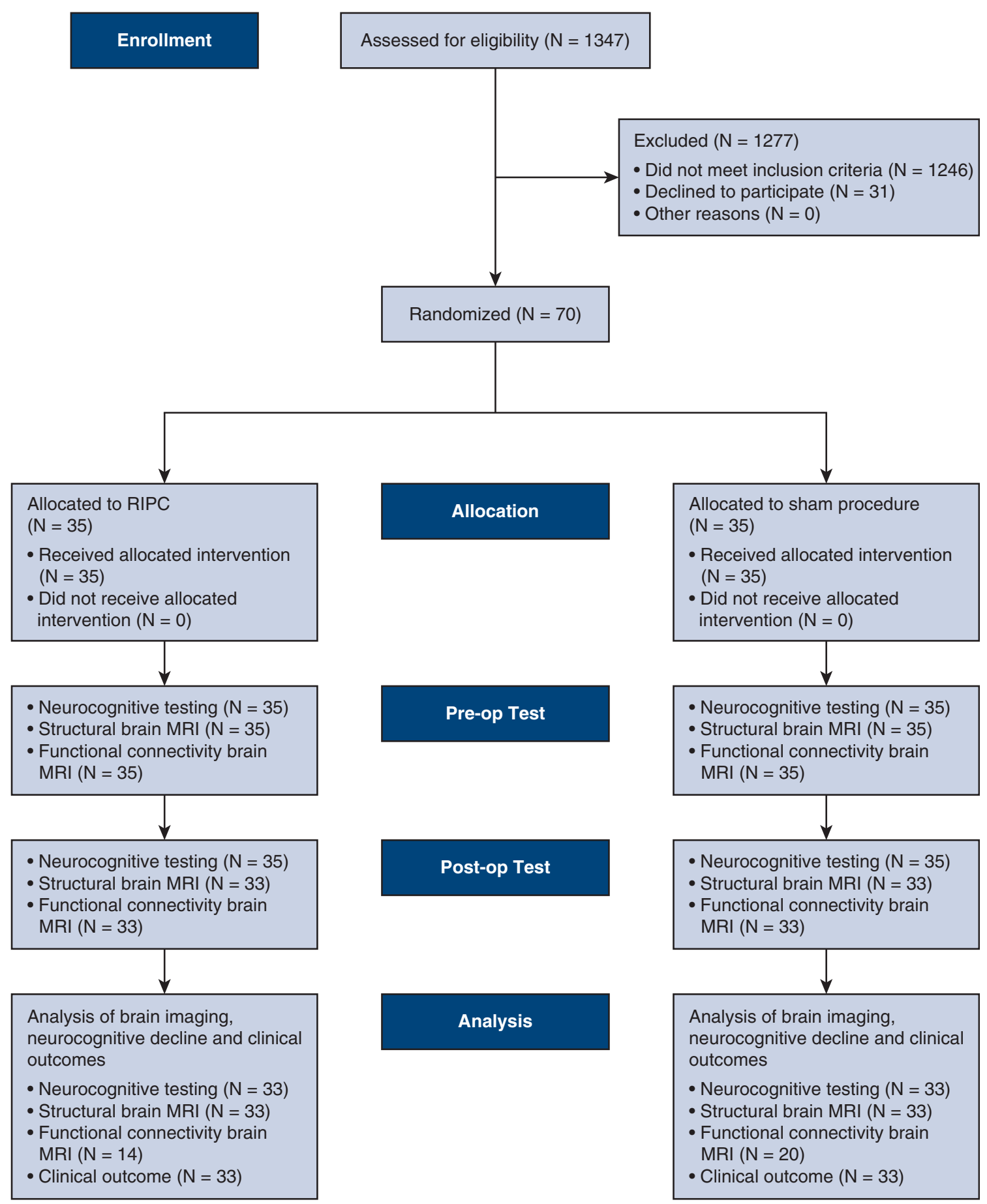

FIGURE E1. Study design flowchart. RIPC, Remote ischemic preconditioning; MRI, magnetic resonance imaging. 
TABLE E1. Localization of new ischemic lesions

\begin{tabular}{lc}
\hline \multicolumn{1}{c}{ Localization } & Number of lesions \\
\hline Right parietal lobe & 2 \\
\hline Right lateral ventricle, occipital horn & 1 \\
\hline Right frontal gyrus & 2 \\
\hline Right cerebellar hemisphere & 2 \\
\hline Periventricular right frontal horn & 1 \\
\hline Right frontal lobe & 9 \\
\hline Right occipitoparietal region & 1 \\
\hline Right temporal lobe & 1 \\
\hline Right splenium & 1 \\
\hline Right occipital region & 1 \\
\hline Right middle frontal gyrus & 2 \\
\hline Left cerebellar hemisphere & 1 \\
\hline Left frontal lobe & 10 \\
\hline Left precentral gyrus & 2 \\
\hline Total & 36 \\
\hline
\end{tabular}

